ARTÍCULO DE REVISIÓN

\title{
EPIDEMIOLOGÍA, USO DE RECURSOS Y COSTOS DE LA ARTRITIS REUMATOIDEA EN ARGENTINA
}

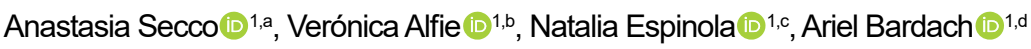 \\ 1 Departamento de Evaluación de Tecnología Sanitaria y Economía de la Salud, Instituto de Efectividad Clínica y Sanitaria \\ (IECS), Buenos Aires, Argentina. \\ a Especialista en Clínica Médica y en Reumatología, maestra en Efectividad Clínica; ${ }^{\text {b }}$ médica especialista en Ortopedia y \\ Traumatología, maestra en Efectividad Clínica; ${ }^{\mathrm{c}}$ magíster en Economía; ${ }^{\mathrm{d}}$ médico especialista en Clínica Médica, doctor en \\ Salud Pública.
}

\section{RESUMEN}

La presente revisión tiene como objetivo identificar información sobre parámetros epidemiológicos y estimar el costo de la artritis reumatoidea (AR) moderada a severa. Se llevó a cabo una búsqueda de la literatura en las principales bases de datos. Se recurrió a un consenso de expertos locales en reumatología para encontrar los parámetros más realistas, utilizando un método Delphi modificado. Se estimaron los costos médicos directos, utilizando información recopilada en la base de datos de costos unitarios del Instituto de Efectividad Clínica y Sanitaria de Argentina. Los costos indirectos se estimaron a través del enfoque del capital humano. Los costos se expresaron en dólares estadounidenses (USD) a noviembre de 2017. La prevalencia reportada de AR en Argentina fue 0,94\% (IC95\%: 0,86 a 1,02), con una tasa de incidencia anual de 19 cada 100000 personas (IC95\%: 17 a 20). El costo anual de las drogas modificadoras de la enfermedad fue de USD 33936,10 por paciente. El costo atribuido a las infecciones serias fue de USD 2474,6. El costo del reemplazo bilateral de rodillas por paciente fue de USD 5276,8, y el del reemplazo total de cadera, de USD 9196,4. El costo por paciente por año de días de hospitalización y los costos indirectos de la AR se acrecentaron al aumentar el puntaje de discapacidad. La revisión reporta información útil acerca de parámetros epidemiológicos y de costos de la AR moderada a severa en la era de los agentes biológicos, con el fin de resultar de utilidad para la conducción de evaluaciones económicas de salud en Argentina.

Palabras claves: Artritis Reumatoide; Personas con Discapacidad; Drogas Antirreumáticas Modificadoras de la Enfermedad; Costos de la Atención en Salud; Argentina (fuente: DeCS BIREME)

\section{EPIDEMIOLOGY, RESOURCE USE AND COSTS OF RHEUMATOID ARTHRITIS IN ARGENTINA}

\begin{abstract}
This review aims to identify information on epidemiological parameters and estimate the cost of moderate to severe rheumatoid arthritis (RA). A search for related literature was carried out in major databases. A consensus of local rheumatology experts was used to find the most realistic parameters, using a modified Delphi method. Direct medical costs were estimated, using information collected from the cost per unit database of the Instituto de Efectividad Clínica y Sanitaria de Argentina. Indirect costs were estimated using the human resources approach. Costs were expressed in US dollars (USD) as of November 2017. The reported prevalence of RA in Argentina was 0.94\% (95\%CI: 0.86 to 1.02), with an annual incidence rate of 19 per 100,000 people (95\%CI: 17 to 20). The annual cost of disease-modifying drugs was 33,936.10 USD per patient. The cost attributed to serious infections was 2,474.6 USD. The cost of bilateral knee replacement per patient was $\$ 5,276.8$ USD; and the cost for total hip replacement was $\$ 9,196.4$. Both, the cost of hospitalization days per patient per year, and the indirect costs of RA increased as the disability score increased. This review reports useful information on epidemiological and cost parameters of moderate to severe RA, in the era of biological agents, in order to be useful for conducting economic evaluations regarding health in Argentina.
\end{abstract}

N, Bardach A. Epidemiología uso de recursos y costos de la artritis reumatoidea en Argentina. Rev Peru Med Exp Salud Publica. 2020;37(3):532-40. doi: https://doi. org/10.17843/rpmesp.2020.373.4766

Correspondencia: Anastasia Secco, asecco@iecs.org.ar

Recibido: $26 / 08 / 2019$ Aprobado: 22/04/2020 En línea: 03/08/2020
Keywords: Arthritis; Rheumatoid; Disabled Persons; Antirheumatic Agents; Health Care Costs; Argentina (Source: MeSH NLM).

\section{INTRODUCCIÓN}

La artritis reumatoidea (AR) es una enfermedad crónica autoinmune que afecta principalmente a las articulaciones y que conduce al daño articular progresivo y a la discapacidad motora. La progresión de la AR puede limitar la capacidad de los pacientes para realizar las 
actividades de la vida diaria y su trabajo ${ }^{(1,2)}$. Actualmente, el tratamiento se basa en el uso de drogas modificadoras de la AR (DMAR), comenzando con las drogas convencionales (cDMAR) frecuentemente metotrexate (MTX) ${ }^{(3)}$. Ante una respuesta inadecuada o intolerancia a una primera y/o segunda línea de tratamiento, existen otras opciones terapéuticas que incluyen diferentes agentes biológicos o drogas modificadoras de la AR biológicas (bDMAR) y pequeñas moléculas ${ }^{(3-6)}$.

Respecto a la epidemiología de la enfermedad en Latinoamérica, las tasas de incidencia y prevalencia varían considerablemente. De acuerdo a un estudio llevado a cabo en diferentes países de América Latina, la prevalencia es cercana al $1 \%{ }^{(7)}$. En un estudio llevado a cabo en México, se observó una prevalencia del 1,6\% (rango: 0,7 a 2,8) ${ }^{(2)}$. En Brasil, un estudio estimó una prevalencia de AR de 0,46\% y otro entre $0,2 \%$ y $1,0 \%{ }^{(8)}$. Otro trabajo realizado en cinco ciudades de Colombia mostró una prevalencia de $0,15 \%$, mientras que en Chile se encontró una prevalencia de 0,46\% (IC95\%: 0,24 a 0,80) ${ }^{(9,10)}$. En Argentina existen escasos datos epidemiológicos provenientes de estudios bien diseñados aunque realizados en poblaciones muy seleccionadas, los cuales podrían no ser lo suficientemente representativos de la realidad de todo el país.

De acuerdo a información del Instituto de Métricas de Salud Estadounidense, en su proyecto de Carga Global de Enfermedad, los años de vida ajustados por discapacidad (AVAD) debido a la AR en Argentina para el 2017 fueron 50,64 por 100000 (IC95\%: 37,93 a 65,01); los casos prevalentes, 304,9 por 100000 habitantes (272,47 a 336,36); y los casos incidentes, 17,29 por 100000 habitantes por año (15,3 a 19,2). Las muertes correspondieron a 0,47 por 100000 habitantes en ese año ${ }^{(11)}$. La prevalencia encontrada es similar a la de Brasil, y ambas más altas que la de México, según esta fuente.

Los costos directos del manejo de la enfermedad están aumentando en los últimos años, a expensas sobre todo de la instauración de los nuevos tratamientos con bDMAR y pequeñas moléculas. A su vez, los costos indirectos secundarios a la discapacidad que acarrea la AR cuando esta no es controlada adecuadamente implican un fuerte impacto económico y una gran problemática de salud pública para todos los países en vías de desarrollo.

El objetivo de esta revisión fue identificar información acerca de los principales parámetros epidemiológicos de la enfermedad y estimar parámetros económicos de la $\mathrm{AR}$ moderada a severa que sirvan como insumos para futuros estudios de costo-efectividad e impacto presupuestario en Argentina. Se estimó además, un probable patrón de uso de recursos en la vida real para el diagnóstico y el tratamiento de la enfermedad.

\section{METODOLOGÍA}

\section{Búsqueda bibliográfica}

Se identificaron los parámetros necesarios para evaluar la carga de la enfermedad basados en las evaluaciones económicas previas, centrados en la variante de AR moderada o severa, ya que representa al paciente promedio. Inicialmente, realizamos una búsqueda exhaustiva de la literatura en las bases de datos electrónicas PubMed, LILACS, CRD (Center for Reviews and Dissemination, University of York), Tripdatabase y sitios web de sociedades científicas. También llevamos adelante una búsqueda genérica en Internet y buscamos manualmente los artículos completos correspondientes a referencias bibliográficas clave. Las estrategias de búsqueda fueron diseñadas por un bibliotecario experto y por el equipo de investigadores para obtener información local e internacional hasta el 20 de noviembre de 2017, sin restricción de idiomas (material suplementario, anexo 1). Los criterios de inclusión para la selección de los estudios fueron estudios observacionales de cohorte, corte transversal, series de casos con al menos 100 pacientes, priorizando la inclusión de publicaciones de la Argentina. En cuanto a las guías de práctica clínica se incluyeron tanto las locales como las internacionales.

\section{Validación con panel Delphi}

En ausencia de evidencia local o cuando los datos provenientes de literatura internacional no eran adaptables a la Argentina, recurrimos a un panel de consenso conformado por expertos locales, utilizando un método Delphi modificado, frecuentemente empleado en casos de ausencia o escasez de información, o en caso que se requiera validar los resultados obtenidos en la literatura. Este método, válido, es una técnica de comunicación estructurada y prospectiva para alcanzar un consenso analizando y reflexionando sobre un problema claramente definido ${ }^{(12,13)}$. De acuerdo con Murphy et al., los grupos de expertos están generalmente conformados por 5 a 20 miembros ${ }^{(14)}$. El equipo de investigación seleccionó un grupo conformado por siete especialistas locales en reumatología, provenientes de diferentes hospitales o centros de reumatología de referencia, tanto del ámbito privado como público, con más de diez años de experiencia en AR en la Argentina. Concurrieron a un encuentro cara a cara y respondieron un cuestionario preespecificado acerca de su opinión sobre la epidemiología de la AR y el uso de recursos en el subgrupo de pacientes de la Argentina, desde una perspectiva social (material suplementario anexo 2). Luego, el equipo de investigación analizó las respuestas para obtener medidas estadísticas simples y resumidas. Finalmente, los investigadores comunicaron las estimaciones a los participantes, para discutir y cambiar su visión, de ser necesario, hasta arribar a un consenso final. 


\section{Estimación de costos}

El costo unitario de cada droga se basó en el precio de venta al público proveniente del Vademecum de la Administración Nacional de Medicamentos, Alimentos y Tecnología Médica (ANMAT), organismo nacional encargado de asegurar la eficacia, seguridad y calidad de los medicamentos, alimentos y tecnologías comercializados en Argentina, además presenta información actualizada de los precios de los medicamentos. El dato fue consultado en noviembre de $2017^{(15)}$. Utilizamos información de precio de venta al público, tasa de uso y cantidades consumidas por año para calcular el costo total de adquisición de las DMAR, desglosando los resultados en cDMAR y bDMAR. Las tasas de uso de cada droga surgieron del panel Delphi modificado y de la literatura, y las dosis consignadas surgen de las recomendaciones de la Sociedad Argentina de Reumatología ${ }^{(4)}$.

Todos los costos médicos directos se estimaron usando el método de microcosteo. Esta técnica consiste en identificar aquellos recursos utilizados durante el diagnóstico, tratamiento y seguimiento de la enfermedad, y en establecer su tasa de utilización, asignándoles valores monetarios. El costo total esperado de cada evento fue el resultado de multiplicar todos los componentes de la ecuación de costo (costo unitario, tasa de uso y cantidades). Los costos unitarios se obtuvieron de la base de costos unitarios del Instituto de Efectividad Clínica y Sanitaria (BCU-IECS) utilizando la perspectiva de seguridad social, información disponible ante el requerimiento ${ }^{(16)}$.

El sistema de salud de la Argentina está dividido en tres subsectores: seguridad social, sector privado y sector público. La seguridad social involucra a las organizaciones centrales del sistema de seguro de salud que abarca la población cubierta por el seguro obligatorio (por ejemplo: contribuciones relacionadas con los ingresos por parte de empleadores y empleados) y cubre alrededor de la mitad de la población argentina ${ }^{(17)}$. Además, utilizamos información de fuentes privadas para estimar algunos costos, expresados en dólares estadounidenses a noviembre de 2017. El tipo de cambio nominal (pesos argentinos/dólares estadounidenses) en noviembre de 2017 fue de 17,31, según los datos del Banco Central de la República Argentina ${ }^{(18)}$.

Los costos de hospitalización fueron presentados en función del cuestionario Health Assessment Questionnaire (HAQ, por sus siglas en inglés) ${ }^{(19)}$. El HAQ es una herramienta ampliamente utilizada para evaluar discapacidad. El cálculo se realizó multiplicando el promedio de días de hospitalización y el costo unitario estimado de un día de hospitalización en sala general desde la perspectiva de la seguridad social ${ }^{(20)}$. Como no se dispone de información local sobre el número anual de días de hospitalización por bandas del puntaje de $\mathrm{HAQ}$, se tomaron los datos reportados por Carlson et al. para Estados Unidos, evaluados en conjunto con el panel Delphi ${ }^{(21)}$. Adicionalmente, se utilizó el micro- costeo para estimar los costos de los eventos adversos. Dado que los eventos adversos corresponden a infecciones, un experto en enfermedades infecciosas identificó el uso esperado de recursos y las cantidades asociadas con cada evento sobre la base de su experiencia y las guías de práctica clínica relevadas. La estimación de los costos incluye el manejo tanto del paciente ambulatorio como del hospitalizado, calculando un promedio de cantidades y tasas de uso.

Los costos indirectos de la AR se estimaron a través del enfoque del capital humano. Este enfoque estima la pérdida de productividad por enfermedad calculando el valor presente, descontado de las ganancias futuras que se esperan en el curso de la enfermedad de la persona. Obtuvimos los datos del salario promedio anual de la base de datos socioeconómica para América Latina y el Caribe para 2017, y los días de trabajo perdidos por HAQ del estudio de Klimes et al. ${ }^{(22,23)}$

\section{SISTEMATIZACIÓN DE RESULTADOS}

\section{Información epidemiológica para Argentina}

Durante el panel Delphi, el grupo de expertos llegó a consenso que la prevalencia estimada por Scublinsky et al. $(0,94 \%$, IC95\%: 0,86 a 1,02) era la más representativa de la situación Argentina, si bien dista de proveer un panorama epidemiológico representativo ${ }^{(24)}$. En este estudio se realizó el cálculo del tamaño muestral basado en el único estudio publicado hasta ese momento respecto de Argentina, teniendo en cuenta que la población de Luján mayor o igual a 16 años era de 53 170, según el Instituto Nacional de Estadísticas y Censos de la República Argentina (INDEC), se calculó un número mínimo de 3742 contactos telefónicos con una distribución por sexo y edad acorde a los parámetros del censo publicados por el INDEC en 2001 y su proyección a 2008. El muestreo se realizó sobre la base de un sistema aleatorizado por computadoras. También estuvieron de acuerdo que la mejor estimación de la tasa de incidencia anual era de 19 cada 100000 personas (IC95\%: 17 a 20), como lo reportó Di et al., en un estudio que incluyó a todos los afiliados al plan de salud de un centro asistencial de referencia del país ${ }^{(25)}$. Estos debían tener al menos un año de seguimiento en dicho centro, ser mayores a 18 años; identificando casos hasta el diagnóstico de AR, la muerte o la finalización del estudio.

De la búsqueda realizada se relevó un único estudio acerca del porcentaje de pacientes con diagnóstico de AR, realizado por un médico en la Argentina. El mismo es el estudio de Mussano et al., el cual incluyó 300 pacientes con AR, quienes contestaron un cuestionario estructurado, en la provincia de Córdoba ${ }^{(26)}$. De acuerdo a este estudio, solo el 53\% había recibido un primer diagnóstico correcto. Este dato fue validado por el panel Delphi como un porcentaje que puede extrapolarse a nivel país. En relación con la severidad de la enfermedad, los expertos también estimaron que el $51 \%$ de los pacientes tendrían AR moderada: el 29\%, severa, y el 20\%, leve. 
De acuerdo a la guía de la Liga Europea contra el Reumatismo (EULAR, por sus siglas en inglés), al Colegio Americano de Reumatología (ACR, por sus siglas en inglés) y a la Sociedad Argentina de Reumatología, se recomienda que los pacientes comiencen tratamiento con CDMAR, siendo el metotrexate el patrón oro ${ }^{(3,4,27)}$. El panel Delphi estimó que un muy bajo porcentaje de pacientes presenta contraindicación absoluta a cDMAR. Por consiguiente, el porcentaje promedio de pacientes que podría iniciar efectivamente tratamiento con CDMAR sería de un 97,3\%. De los cuales, se estima que un $44 \%$ presenta falla a una primera línea de tratamiento con cDMAR, estimación basada en el estudio de Arturi et al., el cual reportó que 44\% de 182 pacientes con AR eran candidatos a tratamiento con agentes biológicos, según guías locales ${ }^{(28)}$. El mismo artículo informó que solo el $11 \%$ de los candidatos a un tratamiento biológico recibía dicha terapia en un hospital público, esto reflejaría los posibles problemas de acceso a biológicos en el sector público del sistema de salud.

Con relación al indicador de cantidad de pacientes que presentan falla a drogas biológicas, el estudio de Kirmayr et al., que incluyó 264 pacientes con AR de la Patagonia Argentina tratados con bDMAR, encontró que el 94\% de ellos fueron tratados con drogas inhibidoras del factor de necrosis tumoral (conocidas como anti-TNF), como el primer bDMAR. El 26\% del total de los pacientes incluidos necesitó un segundo bDMAR, usualmente una anti-TNF (48\%) ${ }^{(29)}$. Con base en estos hallazgos y la búsqueda de literatura internacional, se estimó un porcentaje de pacientes con falla a anti-TNF de aproximadamente $30 \%$ a $50 \%$. Para estimar el porcentaje de pacientes tratados con otros bDMAR, nos basamos en el mismo estudio de Kirmayr et al., que mostró que el 52\% de los pacientes que no respondieron a un primer bDMAR (en 94\% de los casos el primer bDMAR había sido una anti-TNF), recibieron bDMAR diferente a una anti-TNF, siendo las principales opciones: $28 \%$ abatacept, $12 \%$ tocilizumab, y $12 \%$ rituximab.

\section{Costos y uso de recursos}

En los costos médicos directos se incluyen los costos de internación, adquisición y administración de drogas, monito- reo, cirugía de reemplazo articular (rodilla/cadera) y eventos adversos asociados a las drogas. En la Tabla 1 se presentan los resultados de los costos de internación por banda HAQ. Se observa una relación positiva entre los costos de internación por paciente-año y la severidad de la enfermedad expresada mediante el puntaje de banda HAQ. El costo de internación para un paciente con un puntaje de HAQ entre 2,6 y 3 es 20 veces mayor que para un paciente con un puntaje de HAQ entre 0,0 y 0,5 .

La Tabla 2 muestra la frecuencia y el costo unitario de los recursos médicos principales asociadas con el costo de monitoreo de los tratamientos con DMAR. El panel Delphi estimó la frecuencia del uso de los recursos y el costo unitario se estimó desde la perspectiva de seguridad social utilizando la BCU-IECS. El costo de monitoreo fue estimado en USD 749,91 por paciente al año.

En lo que respecta a los costos de administración de las drogas, el costo estimado por infusión fue de USD 104,5 por paciente. Esto incluye el costo de admisión para administración intravenosa de los bDMAR, obtenido mediante la técnica de microcosteo. Por el otro lado, el costo estimado por administración subcutánea fue de USD 14,11 por paciente (en este caso, solo se consideran visitas a enfermería y el material descartable). El costo total de la administración de DMARD por paciente al año es de USD 1205, siendo USD 963,08 el costo total de la administración por infusión intravenosa y USD 241,93, la administración subcutánea, considerando las cantidades consumidas por paciente al año y las tasas de uso y forma de administración de cada droga.

En la Tabla 3 se muestran los costos totales correspondientes a la adquisición de las drogas. El costo total de adquisición de los DMAR se estimó en USD 33936,10 por paciente al año. Este resultado se debe principalmente al costo de los bDMAR. Se identificaron tres eventos adversos de relevancia asociados con los tratamientos para la AR: infección seria, celulitis y herpes zoster (HZ). Asumimos como infección seria a la neumonía con requerimiento de internación. Esta asunción se basó en el metaanálisis de Singh et al. ${ }^{\left({ }^{30}\right)} \mathrm{El}$ costo de este evento se valuó en USD 2474,6. El costo del tratamiento de la celulitis se estimó en USD 1077,1, y del herpes zoster, en

Tabla 1. Número de días de hospitalización y costos de hospitalización por banda de HAQ por paciente-año

\begin{tabular}{|c|c|c|c|c|c|c|}
\hline \multirow{2}{*}{ Bandas HAQ } & \multicolumn{3}{|c|}{ Días de hospitalización (n) } & \multicolumn{3}{|c|}{ Costos hospitalización (USD) } \\
\hline & base & mín. & máx. & base & mín. & máx. \\
\hline $0,0-0,5$ & 0,20 & 0,10 & 0,30 & 29,75 & 14,88 & 44,63 \\
\hline $0,6-1,0$ & 0,40 & 0,30 & 0,50 & 59,50 & 44,63 & 74,38 \\
\hline $1,1-1,5$ & 0,60 & 0,50 & 0,70 & 89,25 & 74,38 & 104,13 \\
\hline $1,6-2,0$ & 0,80 & 0,70 & 0,90 & 119,01 & 104,13 & 133,88 \\
\hline $2,1-2,5$ & 2,00 & 1,80 & 2,20 & 297,52 & 267,76 & 327,27 \\
\hline $2,6-3,0$ & 4,00 & 3,00 & 5,00 & 595,03 & 446,27 & 743,79 \\
\hline
\end{tabular}

HAQ: Health Assessment Questionnaire

Fuente: Panel Delphi, basado en Carlson (2015), y Base de Costos Unitarios de IECS (Instituto de Efectividad Clínica y Sanitaria) 
Tabla 2. Frecuencia y costos de los principales recursos por paciente-año

\begin{tabular}{|c|c|c|c|c|c|}
\hline \multirow[b]{2}{*}{ Recursos } & \multicolumn{3}{|c|}{ Frecuencia mensual } & \multirow{2}{*}{$\begin{array}{l}\text { Costo unitario } \\
\text { (USD) }\end{array}$} & \multirow{2}{*}{$\begin{array}{l}\text { Costo total } \\
\text { (USD) }\end{array}$} \\
\hline & $\begin{array}{l}\text { Al inicio del } \\
\text { tratamiento }\end{array}$ & $\begin{array}{c}\text { Durante los primeros } \\
\text { seis meses }\end{array}$ & $\begin{array}{l}\text { Fase de manteni- } \\
\text { miento }\end{array}$ & & \\
\hline Hemograma completo & 0,89 & 1,83 & 0,25 & 4,07 & 17,16 \\
\hline Eritrosedimentación (ESR) & 0,89 & 1,83 & 0,25 & 1,21 & 5,12 \\
\hline Factor reumatoideo & 0,89 & 0,21 & 0,00 & 19,44 & 21,38 \\
\hline Proteína C reactiva (PCR) & 0,85 & 1,71 & 0,22 & 6,08 & 23,58 \\
\hline Tasa de filtrado glomerular & 0,01 & 0,08 & 0,00 & 1,21 & 0,11 \\
\hline Función renal & 0,89 & 1,72 & 0,25 & 66,60 & 273,72 \\
\hline Función hepática & 0,89 & 1,83 & 0,26 & 70,80 & 303,01 \\
\hline Perfil bioquímico* & 0,89 & 1,83 & 0,25 & 25,08 & 105,83 \\
\hline Total & & & & & 749,91 \\
\hline
\end{tabular}

* Incluye: Test de úrea y creatinina, total proteína, albumina, ácido úrico, colesterol HDL y LDL, triglicéridos.

Fuente: Panel Delphi, basado en la opinión de expertos locales, guías de práctica clínica de la Sociedad Argentina de Reumatología y la base de costos unitarios del (IECS) Instituto de Efectividad Clínica y Sanitaria

USD 1270,4. Este último es consistente con el resultado reportado por Rampakakis et al. ${ }^{(31)}$.

El costo del reemplazo articular se obtuvo de literatura local y actualizado al valor 2017 en pesos argentinos (ARS), basados en el índice de precios al consumidor y luego estimado en USD, con base en el tipo de cambio nominal. El costo médico directo por paciente debido a reemplazo bilateral de rodillas se valuó en USD 5276,8, mientras el costo médico directo por paciente por reemplazo de cadera fue de USD 9196,4 ${ }^{(32,33)}$. Es importante resaltar que ambas estimaciones se basan en el costo de las próstesis fabricadas en la Argentina, las cuales son relativamente más económicas que las de origen extranjero.

Finalmente, en la Tabla 4 se presentan las estimaciones de los costos indirectos de la AR obtenidos a través del enfoque del capital humano para cada banda HAQ. En general, los costos indirectos de la AR por paciente al año aumentan con el puntaje del HAQ, excepto cuando el puntaje del HAQ se encontró en el rango entre 1,1 y 1,6. Los costos indirectos por paciente con un puntaje de HAQ entre 1,6-2,1 es aproximadamente dos veces mayor que los de un paciente con $\mathrm{HAQ}<0,6$.

\section{DISCUSIÓN}

En lo mejor de nuestro conocimiento, esta es la primera revisión en Argentina que evalúa los costos económicos de la AR desde la perspectiva de la seguridad social en términos de tratamiento, monitoreo y costos de eventos adversos en la era de los agentes biológicos, y reúne algunos insumos epidemiológicos importantes para el país. Es así, que nuestro estudio provee información útil para futuras evaluaciones económicas de las drogas y análisis de impacto presupuestario.

No existen en la región ni en Argentina datos representativos poblacionales acerca de indicadores epidemiológicos como prevalencia completa, incidencia anual de casos en los distintos niveles de gravedad ni tasa de letalidad. Los expertos coinciden en que la prevalencia de la AR en Argentina es de aproximadamente $1 \%$, y su tasa de incidencia cercana a 20 por cada 100000 personas. Estos estudios coinciden muy bien con la información provista por el estudio Global Burden of Disease project (GBD), mencionada en la introducción del presente trabajo ${ }^{(11)}$. Esta prevalencia también resulta similar a la reportada en estudios provenientes de Inglaterra, Lituania y América, mientras que la incidencia se encuentra en el rango menor de la tasa global ${ }^{(7,34-36)}$. También es similar a lo reportado por Peláez-Ballesta et al. en 2018, en un estudio que describió una prevalencia de AR de 1,3\% en cuatro poblaciones indígenas de Latinoamérica; sin embargo, en un subanálisis del mismo estudio, la prevalencia en la comunidad indígena Qom de Argentina fue del 2,4\% ${ }^{(37)}$.

La totalidad de la evidencia relevada en la búsqueda bibliográfica fue expuesta al panel Delphi para contar con su aporte para evaluar los resultados de los estudios y, junto con el equipo de investigadores, definir cuáles serían los más representativos de la población argentina. Cabe destacar que nuestro trabajo es un potencial insumo para estudios de análisis presupuestarios y de costo-efectividad, por lo cual incluir datos locales epidemiológicos se considera de relevancia.

Encontramos que más de la mitad de los pacientes con AR moderada a severa presentan falla en la respuesta a $c D$ MAR y requieren tratamiento con bDMAR, siendo las anti-TNF los más frecuentemente utilizados.

Con respecto al porcentaje de pacientes con respuesta inadecuada a las anti-TNF, Favalli et al. publicaron en 2017 una revisión de la literatura mostrando que tanto en los ensayos clínicos aleatorizados (ECA) como en los estudios observacionales, entre $30 \%$ y $40 \%$ de los pacientes tratados con anti-TNF no alcanzan el objetivo terapéutico, no mantienen la buena respuesta inicial a lo largo del tiempo, o experi- 
Tabla 3. Uso de recursos y costos de drogas modificadoras de la artritis reumatoide (DMAR)

\begin{tabular}{|c|c|c|c|c|c|c|}
\hline DMAR & Vía de administración & $\begin{array}{l}\text { Costo } \\
\text { unitario } \\
\text { (USD) }\end{array}$ & Dosificación & Tasa de uso & $\begin{array}{c}\text { Unidades } \\
\text { consumidas por } \\
\text { año } \\
\end{array}$ & $\begin{array}{c}\text { Costo total } \\
\text { por año } \\
\text { (USD) }\end{array}$ \\
\hline Metotrexate & Oral & 1,59 & $15 \mathrm{mg} / \mathrm{semana}$ & $99 \%$ & 48 & 75,36 \\
\hline Etanercept & Subcutáneo $50 \mathrm{mg}$ & 622,77 & $50 \mathrm{mg} / \mathrm{semana}$ & $1 \%$ & 48 & 298,93 \\
\hline $\begin{array}{l}\text { Costo total de primera línea } \\
\text { para drogas convencionales }\end{array}$ & & & & & & 374,29 \\
\hline Etanercept & Subcutáneo $50 \mathrm{mg}$ & 622,77 & $50 \mathrm{mg} / \mathrm{semana}$ & $13 \%$ & 48 & 3850,23 \\
\hline Tofacitinib & Oral & 37,67 & $5 \mathrm{mg} / 2$ comprimidos por día & $6 \%$ & 730 & 1539,83 \\
\hline Abatacept & IV $250 \mathrm{mg}$ & 975,60 & $\begin{array}{l}\text { Pacientes } 60-100 \mathrm{~kg}: \\
\text { Mes 1: } 750 \mathrm{mg} / \mathrm{días} 0,15 \text { y } 30 \\
\text { Mes }+1: 750 \mathrm{mg} / \mathrm{mes}\end{array}$ & $5 \%$ & 42 & 2065,15 \\
\hline Abatacept & Subcutáneo $125 \mathrm{mg}$ & 639,77 & $125 \mathrm{mg} / \mathrm{semana}$ & $3 \%$ & 48 & 1031,82 \\
\hline Adalimumab & Subcutáneo $40 \mathrm{mg}$ & 1192,48 & $40 \mathrm{mg}$ cada 2 semanas & $11 \%$ & 24 & 3045,11 \\
\hline Certolizumab pegol & Subcutáneo $200 \mathrm{mg}$ & 1120,07 & $\begin{array}{l}\text { Mes 1: } 400 \mathrm{mg} / \text { día } 0,15 \text { y } 30 \\
\text { Mes +1: } 400 \mathrm{mg} / \mathrm{mes}\end{array}$ & $6 \%$ & 28 & 1756,27 \\
\hline Golimumab & Subcutáneo $50 \mathrm{mg}$ & 2542,17 & $50 \mathrm{mg} / \mathrm{mes}$ & $3 \%$ & 12 & 854,17 \\
\hline Infliximab & IV $100 \mathrm{mg}$ & 1925,79 & $\begin{array}{c}\text { Paciente } 70 \mathrm{~kg}: 350 \mathrm{mg} / \text { día } 0 \text {, semana } \\
\text { 2, semana 6, } \\
\text { cada } 8 \text { semanas }\end{array}$ & $3 \%$ & 28 & 1509,82 \\
\hline Tocilizumab & IV $200 \mathrm{mg}$ & 979,31 & Paciente $70 \mathrm{~kg}: 560 \mathrm{mg} / \mathrm{mes}$ & $7 \%$ & 36 & 2369,14 \\
\hline Rituximab & IV $500 \mathrm{mg}$ & 4233,32 & $\begin{array}{c}1000 \text { mg/día } 0 \text { y } 15 \text {, re infusión cada } \\
6 \text { meses }\end{array}$ & $1 \%$ & 12 & 284,48 \\
\hline $\begin{array}{l}\text { Costo total de segunda línea } \\
\text { para agentes biológicos }\end{array}$ & & & & & & 18306,03 \\
\hline Abatacept & IV $250 \mathrm{mg}$ & 975,60 & $\begin{array}{c}\text { Paciente } 60-100 \mathrm{~kg}: \\
\text { Mes 1: } 750 \mathrm{mg} / \mathrm{dí} 0,15 \text { y } 30 \\
\text { Mes +1: } 750 \mathrm{mg} / \mathrm{mes}\end{array}$ & $4 \%$ & 42 & 1670,48 \\
\hline Abatacept & Subcutáneo $125 \mathrm{mg}$ & 639,77 & $125 \mathrm{mg} / \mathrm{semana}$ & $5 \%$ & 48 & 1430,79 \\
\hline Rituximab & IV $500 \mathrm{mg}$ & 4233,32 & $\begin{array}{c}1000 \text { mg/ día } 0 \text { y } 15 \text {, re infusión cada } \\
\text { seis meses }\end{array}$ & $4 \%$ & 12 & 2218,94 \\
\hline Tocilizumab & IV $200 \mathrm{mg}$ & 979,31 & Paciente $70 \mathrm{~kg}: 560 \mathrm{mg} / \mathrm{mes}$ & $3 \%$ & 36 & 1231,96 \\
\hline Tocilizumab & Subcutáneo 162 mg & 2663,72 & $\begin{array}{l}\text { Paciente } 100 \mathrm{~kg} \text { o menos: } \\
\qquad 162 \mathrm{mg} / \mathrm{semana}\end{array}$ & $5 \%$ & 48 & 6701,84 \\
\hline Tofacinitib & Oral & 37.67 & $5 \mathrm{mg} /$ dos comprimidos por día & $7 \%$ & 730 & 2001,78 \\
\hline \multicolumn{6}{|c|}{ Costo total de tercera línea bDMAR (falta de respuesta a segunda-línea bDMAR) } & 15255,78 \\
\hline \multicolumn{6}{|c|}{ Costo total de DMAR por paciente-año } & 33936,10 \\
\hline
\end{tabular}

IV: intravenoso

Fuente: Panel Delphi, basado en la opinión de expertos locales, guías de práctica clínica de la Sociedad Argentina de Reumatología y el formulario ANMAT.

mentan eventos adversos, cuyo resultado es la discontinuación del tratamiento ${ }^{(38)}$. En línea con esto, la mayoría de los ECA reportan resultados similares: aproximadamente $40 \%$ de los pacientes no alcanzan la respuesta ACR 20, objetivo mínimo aceptable. En lo que respecta a la remisión clínica medida por respuesta EULAR, la proporción de pacientes clasificados como no respondedores se encuentra entre 50\% y $75 \%$. Estos hallazgos son consistentes con la experiencia poscomercialización reportada por estudios observacionales basados en cohortes de la vida real tratados con anti-TNF, y con registros de Europa y América. Diferentes registros internacionales reportaron una sobrevida de estas drogas a los 5 años de $42 \%$ a $50 \%$, mientras que otros dos registros internacionales observaron una tasa de retención a 3 años de $44,2 \%$ y $52 \%$, respectivamente ${ }^{(38-44)}$. Rolón-Campuzano et al. publicaron en 2018, posterior a la fecha de búsqueda bibliográfica de nuestro trabajo, un estudio llevado a cabo en cinco centros de Argentina, en el que se observó que de 347 pacientes con AR 53,9\% discontinuaron el tratamiento con un primer bDMAR y $41,3 \%$ discontinuaron el tratamiento con el segundo bDMAR. Cabe aclarar que la causa más frecuente de suspensión del primer biológico no fue la falla al tratamiento, sino la falta de provisión; mientras que la del segundo fue la ineficacia ${ }^{(45)}$. 
Tabla 4. Costos indirectos según bandas de HAQ por paciente al año, a través del enfoque del capital humano

\begin{tabular}{lcc}
\hline HAQ & Días perdidos & $\begin{array}{c}\text { Costos indirectos } \\
\text { (USD) }\end{array}$ \\
\hline$<0,6$ & 15 & 206,28 \\
$0,6 \mathrm{a}<1,1$ & 21 & 288,80 \\
$1,1 \mathrm{a}<1,6$ & 17 & 233,79 \\
$1,6 \mathrm{a}<2,1$ & 35 & 481,33 \\
\hline
\end{tabular}

HAQ: Health Assessment Questionnaire

Fuente: SEDLAC (CEDLAS y Banco Mundial), Klimes y col., y BCU IECS

En términos de costos, nuestro estudio mostró que el mayor costo de internación por paciente al año (por ejemplo, USD 595) correspondió a la banda de HAQ de 2,5-3,0 HAQ. El costo total de adquisición de los DMAR fue de USD 33 936,10 y el costo total de la administración de las drogas fue de USD 1205 por paciente al año. Respecto al costo de los eventos adversos, el mayor costo estimado corresponde a las infecciones serias (USD 2475), mientras que el costo del reemplazo articular fue de USD 5277 y USD 9196 para el reemplazo de rodillas y cadera, respectivamente. Observamos que el mayor costo indirecto por paciente-año se encontraba en la banda de HAQ $\geq 1,6-<2,1$ (USD 481).

En 2002, antes de la era de los agentes biológicos, Catay et al. evaluaron el costo de la $\mathrm{AR}$ en un hospital universitario de Argentina ${ }^{(20)}$. Observaron que el 60\% de los costos totales correspondían a costos médicos directos, mientras los costos indirectos representaron un 33\%. Las internaciones representaron el $73 \%$ del total de los costos médicos directos, mientras que los fármacos y los procedimientos ambulatorios simbolizaron el $16 \%$ y el $8 \%$, respectivamente. Sus resultados también muestran que los costos indirectos se incrementan por puntaje de HAQ (variable dicotómica, $\mathrm{HAQ}<0,8$ y HAQ $>0,8)$. Vale la pena señalar que estos datos podrían ser diferentes en la actualidad dado que los mismos se refieren a la era prebiológica. Por otro lado, Tundia et al. estimaron el impacto económico futuro relacionado con la creciente utilización de agentes biológicos para el tratamiento de la AR desde la perspectiva social en Argentina, Brasil, Colombia y México para el periodo 2012-2022 ${ }^{(46)}$. El modelo predijo que la creciente utilización de drogas biológicas para el tratamiento de los pacientes con AR en 2012-2022 resultaría en un ahorro neto acumulado de ARS 2,3 billones en 2012, en Argentina. Los costos indirectos estimados compensarían a aquellos asociados con el uso extensivo de agentes biológicos.

Una de las fortalezas de la presente revisión es el haber estimado los costos por bandas de HAQ, adaptando información internacional al contexto local y validando los datos con un panel Delphi. Los resultados mostraron significativa variación en los costos de internación y los costos indirectos por bandas de HAQ. Encontramos que a mayor puntaje de
HAQ, mayores los costos asociados con el diagnóstico o el tratamiento. El consenso de expertos puede proveer valiosa información, particularmente en los casos en los que la evidencia es limitada. Los métodos formales de consenso por expertos, como el panel Delphi utilizado en este estudio, mejoran la responsabilidad y la transparencia de los procesos de toma de decisiones, ayudan a reducir los sesgos y el uso de heurísticas, y proporcionan una estructura que permite expresar incertidumbre. En 2017, Bojke et al. publicaron una guía acerca del Proceso de Generación de Prioridades de Consenso. Afirmaron que además de consensuarse probabilidades u ocurrencias de eventos, es posible validar costos, el valor de la calidad de vida y la efectividad relativa ${ }^{(47)}$. Se prefieren los encuentros cara a cara entre los expertos y este fue el enfoque elegido en nuestro estudio.

La estimación de los costos directos e indirectos nos permitió conocer la carga económica total de la enfermedad, incluyendo los costos médicos enfrentados por los proveedores de atención médica y el costo de oportunidad para los pacientes (pérdida de ingresos debido a la enfermedad) desde una perspectiva social. Esto proporciona información para la toma de decisiones dentro del sistema de salud a nivel general (macroeconómico). Por lo tanto, contabilizar los costos directos e indirectos es otra de las fortalezas de nuestro estudio.

Sin embargo, nuestra revisión tiene algunas limitaciones. La estimación de los parámetros epidemiológicos presenta problemas inherentes al tipo de datos y puede no representar la situación de todo el país. Otra limitación podría ser que algunos de los estudios seleccionados incluyen poblaciones mixtas (pacientes internados y ambulatorios). Para superar esta limitación, seleccionamos la metodología Delphi con el fin de validar las estimaciones, la cual es una herramienta útil frecuentemente utilizada en estos casos. Es importante mencionar que el análisis de costos se realizó desde una perspectiva de seguridad social, en un sistema de salud fragmentado como el argentino, el cual cubre cerca de la mitad de la población. En un estudio que explora la base de datos de los costos unitarios utilizada en este estudio por subsector, se observa que la seguridad social tiene un costo intermedio entre el sector público y el sector privado, si a esto le sumamos el importante peso relativo de este subsector, se puede considerar que la estimación realizada en este estudio es una buena aproximación del comportamiento del costo en el sistema de salud argentino.

\section{CONCLUSIÓN}

Nuestra revisión recolecta y estima parámetros epidemiológicos y costos relevantes relacionados con la AR moderada a severa, una enfermedad que impone una considerable carga en los países en desarrollo. Este es un primer acercamiento para futuras evaluaciones de costo-efectividad y análisis de impacto presupuestario que analicen las tecnologías de alto costo en Argentina. 
Agradecimientos: Queremos agradecer a Ulises Garay (MSc en Economía) por su contribución en el proyecto, así como la participación de todos los miembros del panel Delphi (en orden alfabético): Cecilia Asnal MD, Gustavo Casado MD, Tomas Cazzenave MD, Damián Duarte Noe MD, Fernando Sommerfleck MD y Javier Rosa MD. Como siempre, valoramos la asistencia de nuestro bibliotecario, Daniel Comandé, y de nuestra asistente administrativa, Gabriela Rodríguez.

Contribuciones de los autores: Todos los autores contribuyeron en la realización del estudio, y en la redacción y revisión del manuscrito.

\section{REFERENCIAS BIBLIOGRÁFICAS}

1. Hu H, Luan L, Yang K, Li SC. Burden of rheumatoid arthritis from a societal perspective: A prevalence-based study on cost of this illness for patients in China. Int J Rheum Dis. 2018;21(8):1572-80.

2. Ramírez LA, Rodríguez C, Cardiel MH. Burden of illness of rheumatoid arthritis in Latin America: a regional perspective. Clin Rheumatol. 2015;34(S1):9-15.

3. Smolen JS, Landewé R, Bijlsma J, Burmester G, Chatzidionysiou K, Dougados $\mathrm{M}$, et al. EULAR recommendations for the management of rheumatoid arthritis with synthetic and biological disease-modifying antirheumatic drugs: 2016 update. Ann Rheum Dis. 2017;76(6):960-77.

4. Sociedad Argentina de Reumatología. Actualización de las guías de práctica clínica en el tratamiento de la artritis reumatoidea. Rev Argent Reumatol. 2008;19(3): 5-9.

5. Alemao E, Johal S, Al MJ, Rutten-Van Mölken M. Cost-Effectiveness Analysis of Abatacept Compared with Adalimumab on Background Methotrexate in Biologic-Naive Adult Patients with Rheumatoid Arthritis and Poor Prognosis. Value Health. 2018;21(2):193-202. doi: 10.1016/j.jval.2017.05.020.2018.

6. Chermont GC, Kowalski SC, Ciconelli RM, Ferraz MB. Resource utilization and the cost of rheumatoid arthritis in Brazil. Clin Exp Rheumatol. 2008;26(1):24-31.

7. Rudan I, Sidhu S, Papana A, Meng S-J, Xin-Wei Y, Wang W, et al. Prevalence of rheumatoid arthritis in low-and middle-income countries: A systematic review and analysis. J Glob Health. 2015;5(1):010409.

8. Oliveira Junior HA de, Almeida AM, Acurcio FA, Santos JB dos, Kakehasi AM, Alvares J, et al. Profile of patients with rheumatic diseases undergoing treatment with anti-TNF agents in the Brazilian Public Health System (SUS), Belo Horizonte - MG. Brazilian J Pharm Sci. 2015;51(3):709-19.

9. Machado-Alba JE, Ruiz AF, Medina Morales DA. The epidemiology of rheumatoid arthritis in a cohort of Colombian patients. Rev Colomb Reumatol. 2015;22(3):148-52.

10. Guía Clínica AUGE. Artritis reumatoide. Serie guías clínicas MINSAL Chile [Internet]. Ministerio de Salud; 2014 [cited 2018 Oct 16]. Available from: http://www.repositoriodigital.minsal.cl/handle/2015/528.

11. University of Washington. GBD Compare. IHME Viz Hub [Internet]. Seattle: University of Washington; 2017 [cited 2020 Mar 2]. Available from: https://vizhub.healthdata.org/gbd-compare/.

12. Varela-Lema L, Atienza-Merino G, López-García M. Priorización de intervenciones sanitarias. Revisión de criterios, enfoques y rol de las agencias de evaluación. Gac Sanit. 2017;31(4):349-357. doi: 10.1016/j. gaceta.2016.09.015.

13. Davis JH. Some compelling intuitions about group consensus decisions, theoretical and empirical research, and interpersonal aggregation phenomena: Selected examples 1950-1990. Organ Behav Hum Decis Process. 1992;52(1):3-38.

14. Murphy M. Consensus development methods and their use in clinical guideline development. Health Technol Assess. 1998;2(3):1-88.

15. Ministerio de Salud de Aragentina. Vademécum Nacional de Medicamentos (VNM) [Internet]. Buenos Aires: Ministerio de Salud; 2017 [cited 2020 Mar 2]. Available from: https://servicios.pami.org. $\mathrm{ar} / \mathrm{vademecum} /$ views/consultaPublica/listado.zul.
Todos aprobaron la versión final y asumen responsabilidad de los contenidos.

Fuentes de financiamiento: Este trabajo de investigación fue financiado por Eli Lilly, a través de un subsidio irrestricto. El patrocinador no tuvo ningún papel en el desarrollo del manuscrito.

Conflictos de interés: Los autores declaran no tener ningún conflicto de interés.

Material suplementario: Disponible en la versión electrónica de la RPMESP.

16. Departamento de Evaluación de Tecnologías Sanitarias y Economía de la Salud. Instituto de Efectividad Clínica y Sanitaria (IECS). Consorcio de costos sanitarios, análisis de impacto presupuestario, y evaluaciones económicas de tecnologías sanitarias [Internet]. Buenos Aires: IECS [cited 2020 Mar 2]. Available from: https://www.iecs.org.ar/consorcio-de-analisis-de-impacto-presupuestario-aip-y-base-de-costos-sanitarios-bcs/.

17. Instituto Nacional de Estadística y Censos (INDEC). Indicadores de salud. Censo Nacional de Población 2010 [Internet]. Buenos Aires: INDEC [cited 2020 Mar 2]. Available from: https:/www.indec.gob.ar/.

18. Banco de la Nación Argentina [Internet]. Buenos Aires: Banco de la Nación [cited 2018 Dec 5]. Available from: http://www.bna.com.ar/ Personas.

19. Fries JF, Spitz PW, Young DY. The dimensions of health outcomes: the health assessment questionnaire, disability and pain scales. J Rheumatol. 1982;9(5):789-93.

20. Soriano E, Catay, Castel del Cid, Narvaez, Velozo, Rosa J, et al. Cost of rheumatoid arthritis in a selected population from Argentina in the prebiologic therapy era. Clin Outcomes Res. 2012;4:219.

21. Carlson JJ, Ogale S, Dejonckheere F, Sullivan SD. Economic evaluation of tocilizumab monotherapy compared to adalimumab monotherapy in the treatment of severe active rheumatoid arthritis. Value Health. 2015;18(2):173-9.

22. Universidad de la Plata, Socio-Economic Database for Latin America and the Caribbean (CEDLAS and The World Bank). Monthly labor income in nominal LCU by gender, age, education and area [Internet]. La Plata: Universidad de la Plata; 2017 [cited 2020 Mar 2]. http://www. cedlas.econo.unlp.edu.ar/wp/en/estadisticas/sedlac/.

23. Klimeš J, Vocelka M, Šedová L, Doležal T, Mlčoch T, Petř́íková A, et al. Medical and productivity costs of rheumatoid arthritis in The Czech Republic: cost-of-illness study based on disease severity. Value Heal Reg Issues. 2014;4(1):75-81.

24. Scublinsky D, Venarotti H, Citera G, Messina OD, Scheines E, Rillo $\mathrm{O}$, et al. The Prevalence of Rheumatoid Arthritis in Argentina. JCR J Clin Rheumatol. 2010;16(7):317-21.

25. Di WT, Vergara F, Bertiller E, Gallardo MA, Gandino I, Scolnik M, et al. Incidence and Prevalence of Rheumatoid Arthritis in a Health Management Organization in Argentina: A 15-year Study. J Rheumatol. 2016;43(7):1306-11.

26. Mussano E, Missakian S, Onetti CM, Mussano CE. Estudio demográfico-social de pacientes con artritis reumatoidea en la provincia de Córdoba (Argentina). Rev Argent Reumatol. 2007;18(4):23-7.

27. Singh JA, Saag KG, Bridges SL, Akl EA, Bannuru RR, Sullivan MC, et al. 2015 American College of Rheumatology Guideline for the Treatment of Rheumatoid Arthritis. Arthritis Rheumatol. 2016;68(1):1-26.

28. Arturi P, Orazio AD', Citera G, Cocco JAM. Indicación de terapia biológica en pacientes con enfermedades reumáticas de la consulta ambulatoria. Rev Argent Reumatol. 2008;19(1):34-8.

29. Kirmayr K, Garibotti G. Patrones de Tratamiento con Agentes Biológicos en Pacientes con Artritis Reumatoidea: Asociación con su sobrevida. Rev Argent Reumatol [Internet]. 2017;Supl 1:20 [cited 2020 
Mar 2]. Available from: http://revistasar.org.ar/revistas/2017/50_congreso_argentino_reumatologia.pdf.

30. Singh JA, Cameron C, Noorbaloochi S, Cullis T, Tucker M, Christensen $\mathrm{R}$, et al. Risk of serious infection in biological treatment of patients with rheumatoid arthritis: A systematic review and meta-analysis. Lancet. 2015;386(9990):258-65.

31. Rampakakis E, Pollock C, Vujacich C, Toniolo Neto J, Ortiz Covarrubias A, Monsanto $\mathrm{H}$, et al. Economic Burden of Herpes Zoster ("culebrilla") in Latin America. Int J Infect Dis. 2017;58:22-6.

32. Mana Pastrián D, Garabano GG, Nazur G, Pesciallo CC, Vedoya S, Viale GG, et al. Reemplazo total de rodilla bilateral: Análisis de costos entre uno y dos tiempos. Rev Asoc Argent Ortop Traumatol. 2010;75(2):151-6.

33. Agnes E, Ávila E, Breme L, Damore GA, Donaire D, Pablo JM, et al. Reemplazo total de cadera. Análisis de la relación costo/beneficio entre el uso de prótesis importadas y prótesis nacionales. Monografía del Curso de Auditoría 2013 [Internet]. Hospital Alemán; 2013 [cited 2018 Oct 24]. Available from: http://www.auditoriamedicahoy.com. ar/biblioteca/Reemplazo\%20total\%20de\%20cadera.\%20Costos\%20 y\%20beneficios\%202013.pdf.

34. Hunter TM, Boytsov NN, Zhang X, Schroeder K, Michaud K, Araujo AB Prevalence of rheumatoid arthritis in the United States adult population in healthcare claims databases, 2004-2014. Rheumatol Int. 2017;37(9):1551-7.

35. Symmons D, Turner G, Webb R, Asten P, Barrett E, Lunt M, et al. The prevalence of rheumatoid arthritis in the United Kingdom: new estimates for a new century. Rheumatology. 2002;41(7):793-800.

36. Adomaviciute D, Pileckyte M, Baranauskaite A, Morvan J, Dadoniene J, Guillemin F. Prevalence survey of rheumatoid arthritis and spondyloarthropathy in Lithuania. Scand J Rheumatol. 2008;37(2):113-9.

37. Peláez-Ballestas I, Granados Y, Quintana R, Loyola-Sánchez A, Julián-Santiago F, Rosillo C, et al. Epidemiology and socioeconomic impact of the rheumatic diseases on indigenous people: An invisible syndemic public health problem. Ann Rheum Dis. 2018;77(10):13971404.

38. Favalli EG, Raimondo MG, Becciolini A, Crotti C, Biggioggero M, Caporali R. The management of first-line biologic therapy failures in rheumatoid arthritis: Current practice and future perspectives. Autoimmun Rev. 2017;16(12):1185-95.

39. Sarzi-Puttini P, Antivalle M, Marchesoni A, Favalli EG, Gorla R, Filippini $\mathrm{M}$, et al. Efficacy and safety of anti-TNF agents in the Lombardy rheumatoid arthritis network (LORHEN). Reumatismo. 2008;60(4):290-5. doi: 10.4081/reumatismo.2008.290.

40. Dennison EM, Packham J, Hyrich K. The BSRBR-RA at 15 years. Rheumatology. 2016;55(12):2093-5.

41. de Punder YMR, Fransen J, Kievit W, Houtman PM, Visser H, van de Laar MAFJ, et al. The prevalence of clinical remission in RA patients treated with anti-TNF: results from the Dutch Rheumatoid Arthritis Monitoring (DREAM) registry. Rheumatology. 2012;51(9):1610-7.

42. Eriksson JK, Askling J, Arkema E V. The Swedish Rheumatology Quality Register: optimisation of rheumatic disease assessments using registred-enriched data. Clin Exp Rheumatol. 2014;32(5 Suppl 85):S-147-9.

43. Gruppo Italiano Studio Early Arthriti [Internet]. GISEA [cited 2018 Oct 24]. Available from: http://www.gisea.eu/.

44. Gabay C, Riek M, Scherer A, Finckh A, SCQM collaborating physicians. Effectiveness of biologic DMARDs in monotherapy versus in combination with synthetic DMARDs in rheumatoid arthritis: data from the Swiss Clinical Quality Management Registry. Rheumatology. 2015;54(9):1664-72.

45. Rolón Campuzano R, Dal Pra F, Schneeberger E, Coronel Ale A, Cerda $\mathrm{O}$, Correa $\mathrm{M}$, et al. Patrones de tratamiento con agentes biológicos: Eficacia y sobrevida a largo plazo en pacientes con artritis reumatoidea. Rev Argent Reumatol. 2018;29(4):14-9.

46. Tundia N, Kotze PG, Rojas Serrano J, Mendes de Abreu M, Skup $\mathrm{M}$, Macaulay D, et al. Economic impact of expanded use of biologic therapy for the treatment of rheumatoid arthritis and Crohn's disease in Argentina, Brazil, Colombia, and Mexico. J Med Econ. 2016;19(12):1187-99.

47. Bojke L, Grigore B, Jankovic D, Peters J, Soares M, Stein K. Informing Reimbursement Decisions Using Cost-Effectiveness Modelling: A Guide to the Process of Generating Elicited Priors to Capture Model Uncertainties. Pharmacoeconomics. 2017;35(9):867-77. 